\title{
High Rate of Adherence to Urate-Lowering Treatment in Patients with Gout: Who's to Blame?
}

Fernando Perez-Ruiz (D) · Nuria Perez-Herrero · Pascal Richette •

Austin G. Stack

Received: September 28, 2020 / Accepted: October 19, 2020 / Published online: October 28, 2020

(c) The Author(s) 2020

\section{ABSTRACT}

Introduction: Gout is commonly associated with low adherence rates, thus limiting the effectiveness of treatment. Nevertheless, informed and empowered patients may be more likely to achieve high adherence. We intend to demonstrate that adherence in clinical practice may reach that achieved in clinical trials.

F. Perez-Ruiz ( $\square)$

Rheumatology Division, Osakidetza, OSI-EE Cruces, Cruces University Hospital, Barakaldo, Spain e-mail: fernando.perezruiz@osakidetza.eus

\section{F. Perez-Ruiz}

BioCruces-Bizkaia Health Research Institute, Barakaldo, Spain

\section{F. Perez-Ruiz}

Department of Medicine, Medicine and Nursing School, University of the Basque Country, Biscay," Spain

\section{N. Perez-Herrero}

Medicine and Nursing School, University of the Basque Country, Biscay, Spain

P. Richette

Rheumatology Department and Inserm URM 1132, Centre Viggo Petersen, Hôpital Lariboisière (AP-HP), and Université Paris Diderot, USPC, Paris, France

\section{A. G. Stack}

Nephrology Division, Health Research Institute, Graduate Entry Medical School, University Hospital Limerick, University of Limerick, Limerick, Ireland
Methods: This was a transversal study within an inception cohort of patients with gout prospectively followed up. Patients were informed at entrance in the cohort of outcomes, targets, and means to implement for successful treatment. Adherence was evaluated through electronic medication possession ratio (MPR) for urate-lowering medication and oral medications for hypertension, diabetes, and hyperlipidemia for comparison. Factors associated with nonadherence, and the relation between nonadherence and serum urate levels while on treatment were analyzed.

Results: Data were retrieved from 336 patients, who showed a mean MPR of $87.5 \%$, with $82.1 \%$ of patients showing $\mathrm{MPR} \geq 0.8$. Rates of adherence for hypertension, hyperlipidemia, and diabetes were quite similar $(88 \%, 87 \%$, and $83 \%$, respectively), although MPR $>0.8$ was significantly lower for oral medications for diabetes. Adherence was lower, but nevertheless quite fair, during the first year of follow-up, and increasing over time. Active follow-up and comorbidity were associated with good adherence, and adherence and long-term follow-up were associated with higher rates of achieving serum urate within therapeutic target.

Conclusion: Patients with gout show high rates of adherence if empowered. Active follow-up and comorbidity are associated with high rates of adherence. Adherence is strongly associated with higher rates of achievement of therapeutic serum urate target. 
Keywords: Adherence; Gout; Treatment

\section{Key Summary Points}

Adherence to treatment in gout is commonly low, declining with time on prescription.

Patient education initially is associated with high rates of adherence and low rates of loss to clinical follow-up.

Failure of treatment due to nonadherence is associated with increase burden of disease and costs, whereas improving adherence is cost-effective.

\section{DIGITAL FEATURES}

This article is published with digital features, including a summary slide, to facilitate understanding of the article. To view digital features for this article go to https://doi.org/10.6084/ m9.figshare.13109675.

\section{INTRODUCTION}

Gout is considered a chronic disease and is the most common cause of inflammatory arthritis in adults. Effective control of gout will likely require long-term, if not life-long, prescription of medications [1]. Therefore, adherence to long-term medication, namely urate-lowering therapy (ULT), is essential for effective control of gout as nonadherence is associated with both poorer clinical outcomes and increased costs [2].

Overall adherence rates for several chronic diseases are lower than 50\%, and are associated with poor health outcomes and increased costs, with significant pharmacoeconomic implications [3]. Gout is considered a paradigmatic example of nonadherence [4]. For example, the rate of adherence to allopurinol in a developed country was reported to be $45.9 \%$ at month 1 ,
$16.7 \%$ at month $3,10 \%$ at month 6 , and an abysmal 3.2\% at month 12 [5].

Barriers to adherence are diverse, from prescribers who may mistrust patients to patients who feel stigmatized by the diagnosis [6]. Patients with chronic conditions are to be supported, not blamed [7], as empowerment of patients participating in clinical trials generally leads to very high rates of adherence both during the trial and in the longer term after its completion [8].

In this study we sought to demonstrate the impact of patient education and patient empowerment on follow-up on adherence rates to ULT in routine clinical practice, and to assess whether these were similar to that reported for randomized clinical trials [9].

\section{METHODS}

\section{Design}

This was a transversal study within a nested cohort of patients with gout who are prospectively followed up. This study was conducted in accordance with the Helsinki Declaration. Data were retrieved from a gout clinical database that is approved by the local ethics committee (Cruces University Hospital Ethics and Investigation Committee), with informed-signed permission from patients or relatives if appropriate.

\section{Population}

Patients with a scheduled follow-up visit from January 2, 2016 to December 24, 2019 and with a prescription for ULT were selected for inclusion in the cohort. The attendance record of all patients at clinic appointments was captured prospectively including lost to follow-up. Patients with a follow-up of less than 3 months were not included.

The rheumatology program is located in a hospital-based facility in a university hospital with a reference population close to half a million people at the time of the study.

At cohort inception, the investigator (FPR) gave the patients and caregivers, if appropriate, 
information on the diagnosis, severity of the disease, proposed targets (flares, serum urate levels), timeline of outcomes (flares, tophi), as well as specific interventions (medications for urate-lowering, prophylaxis, and flares). Such information was reinforced during clinical visits in the first year of therapy, typically at 3,6 , and 12 months of follow-up, but no further contact was provided to patients between scheduled clinical visits. The number of visits is usually two per year during the second year, and once a year thereafter (twice for patients with severe disease or complex patients). The patients were unaware of the existence of a tracking system for adherence, although they were informed afterwards if needed after data were obtained.

\section{Variables and Statistical Analysis}

General data (age, sex, comorbid conditions, previous treatment) along with gout-related variables (time from onset, number of flares in the previous year, number of joints ever involved, X-ray results, treatments and doses prescribed for gout, baseline and follow-up serum urate) are systematically obtained at entry into the cohort (first clinical visit). Therapeutic target serum urate (sUA) was considered to be below $0.36 \mathrm{mmol} / \mathrm{L}$ (less than $6 \mathrm{mg} / \mathrm{dl}$ ) [10]. Diabetes, hypertension, and hyperlipidemia were defined as per definitions at the time of cohort entry or being prescribed specific medications for each of them. Previous cardiovascular event was defined as in the Anti-Platelet Trialist Collaboration (APTC) definitions [11]. Significant alcohol intake was considered when consumption exceeded $15 \mathrm{~g} /$ day. Comorbidity was evaluated using the Kaiser Permanente pyramid strata that are provided by the Health Department in the electronic file of the patients [12]. Patients classified into the highest stratum were considered as complex patients.

Adherence was assessed using information retrieved from government-provided electronic pharmacy databases that covered more than $98 \%$ of the population and is expressed as the medication possession ratio (MPR). MPR $\geq 0.80$ is considered to be good, fair for values ranging from 0.60 to 0.79 , and low if values are less than 0.60. The electronic system shows an alarm signal in the prescription panel for patients whose medications have an MPR $<0.80$ of the prescribed dose. MPR data are expressed as the average prescribed dose during the previous year of prescription, excluding in-hospital care periods if they occurred within the Basque Country. The treatment of patients who die or live outside the Basque Country cannot be retrieved in the system. Adherence was determined primarily for patients who were prescribed urate-lowering medications, but also antihypertension treatments, oral hypoglycemic medications for diabetes, and lipidlowering agents as a comparison.

Analysis of adherence data was determined at the time of scheduled follow-up visits and also at the following time periods: 1st, 2nd, 3rd year of follow-up and beyond 3 years of followup. Multivariable models were constructed to determine factors associated $(p<0.2)$ with an MPR $<0.8$. Statistical analysis was performed using the statistical package SPSS23.

\section{RESULTS}

There were 358 patients who had scheduled follow-up clinic appointments during the total observation period. Of these, 11 died (treatment data no longer available in the electronic file) and nine had no MPR data because of their place of residence outside the Basque Country, leaving a total of 336 patients with follow-up data during the study period that fulfilled criteria for inclusion. The diagnosis of gout was based on microscopy of synovial fluid samples from joints or material aspirated from tophi in $78.5 \%$ of patients, on ultrasound specific findings in $14.9 \%$, and based on clinical manifestations alone in $6.6 \%$. Less than $50 \%$ of all patients had ever been prescribed any uratelowering medication before baseline visit at rheumatology.

The baseline characteristics of the overall cohort are shown in Table 1 . Twenty-seven $(8.0 \%)$ patients did not attend the scheduled clinic visits and were lost to follow-up. Loss to follow-up occurred more frequently during the 
Table 1 General characteristics of the patients

\begin{tabular}{lllll}
\hline Variable & All $(\boldsymbol{N}=\mathbf{3 3 6})$ & MPR $\geq \mathbf{0 . 8}(\boldsymbol{N}=\mathbf{2 7 6})$ & MPR $<\mathbf{0 . 8}(\boldsymbol{N}=\mathbf{6 0})$ & $\boldsymbol{p}$ \\
\hline Age (years) & $62.2 \pm 14.2$ & $62.7 \pm 14.2$ & $59.6 \pm 14.7$ & 0.125 \\
Sex (\% male) & 89.6 & 91.7 & 84.0 & 0.091 \\
Time from onset (years) & $7 . \pm 8.3$ & $7.2 \pm 8.6$ & $7.7 \pm 6.7$ & 0.721 \\
Complex patients (\%) & 23.8 & 27.2 & 4.54 & 0.072 \\
Polyarticular (\%) & 41.1 & 43.9 & 38.0 & 0.520 \\
Subcutaneous tophi (\%) & 40.8 & 39.8 & 46.7 & 0.326 \\
Follow-up (months) & $45.7 \pm 53.8$ & $46.9 \pm 54.7$ & $34.8 \pm 48.5$ & 0.115 \\
Flares per year & $4.2 \pm 5.9$ & $4.5 \pm 6.1$ & $4.2 \pm 5.0$ & 0.774 \\
No previous ULT (\%) & 46.6 & 46.1 & 49.2 & 0.961 \\
Ethanol > 15 g/day (\%) & 27.0 & 27.6 & 24.1 & 0.589 \\
Hypertension (\%) & 64.1 & 66.4 & 53.4 & 0.062 \\
Diabetes (\%) & 19.4 & 19.9 & 17.2 & 0.649 \\
Hyperlipidemia (\%) & 58.8 & 57.6 & 51.0 & 0.539 \\
Previous CV event (\%) & 37.3 & 37.9 & 34.5 & 0.619 \\
Lost to follow-up (\%) & 8.0 & 4.7 & 23.3 & $<0.001$ \\
sUA < 6 mg/dl (\%) & 87.5 & 90.9 & 71.6 & $<0.001$ \\
\hline
\end{tabular}

first year $(17 / 126,13.5 \%)$ than during the 2 nd or 3rd year of follow-up (5/92, 5.4\%) or beyond the 3rd year $(5 / 118,4.2 \%)$, results being statistically significant $(p<0.01)$. Nonattendance at scheduled visits was far more common in nonadherent patients than for those who adhered to ULT (23.3\% vs $4.7 \%$ respectively).

Overall adherence to treatment at the time of evaluation was $87.5 \%$ for ULT (median 92.5, interquartile range $84-98$ ), while it was good (MPR > 0.8) in 82.1\%, fair (MPR 0.6-0.79) in $11.0 \%$, and poor (MPR $<0.6$ ) in $6.8 \%$. Adherence to ULT was in general very high, and similar to that for medications prescribed for hypertension (224 patients, 66.7\%) and hyperlipidemia (131, 39\%), but substantially higher than that for oral medications for diabetes (47 patients, 14\%), as shown in Fig. 1. Good adherence seemed to be associated in bivariate analysis (Table 1) with aging, male gender, longer time of follow-up, taking medications for hypertension, attending scheduled visits, and patient's complexity. sUA in therapeutic target (less than $6 \mathrm{mg} / \mathrm{dl}$ ) was associated in bivariate analysis (data not shown) with good adherence, time from onset, polyarticular joint involvement from onset, time on follow-up, male gender, lower baseline sUA, and presence of chronic kidney disease (CKD).

Analysis of adherence rates at 1 year, 2 years, and beyond 2 years of follow-up revealed that $74.6 \%, 87 \%$, and $86.4 \%$ of patients, respectively, achieved MPR $>0.8$, which were significantly different for the latter years compared to year 1 (Fig. 2).

Multivariable regression analysis showed that presence of comorbidity, attending scheduled visits, and male gender are independently and statistically associated with good adherence, while adherence and time on follow-up are statistically associated with reaching target sUA $<6 \mathrm{mg} / \mathrm{dl}$ (Tables 2 and 3 ). 


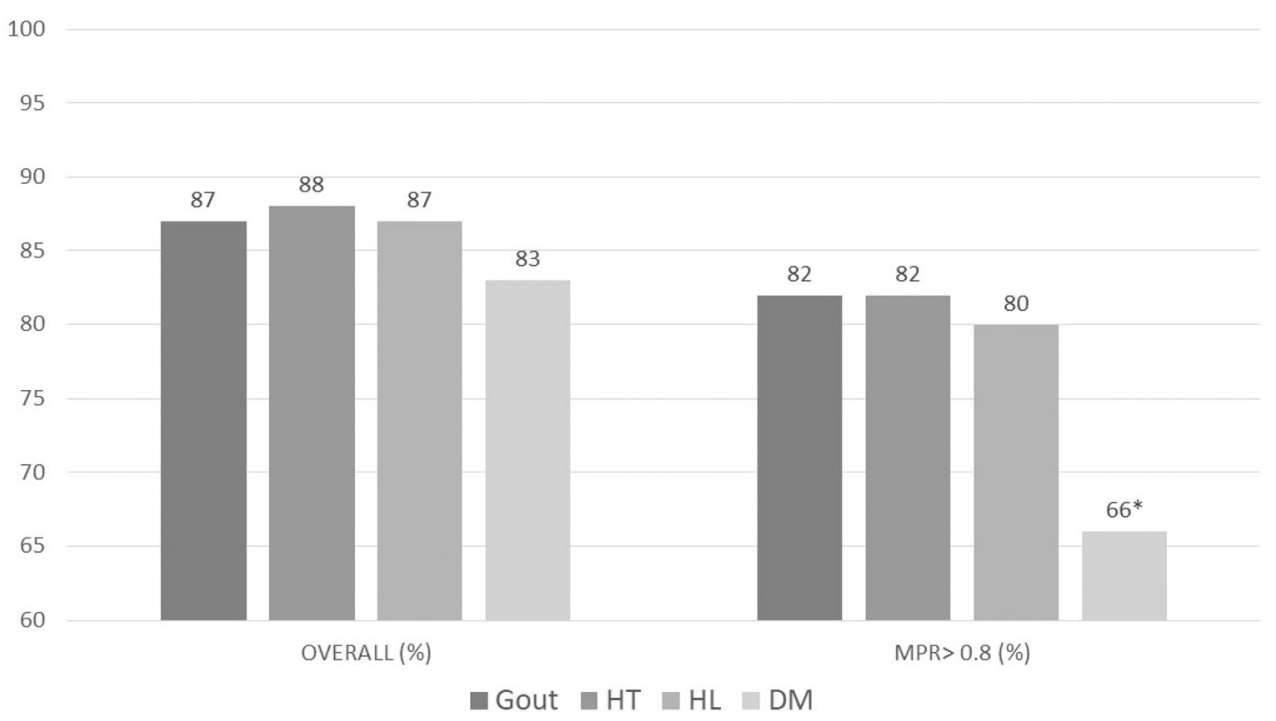

Fig. 1 Adherence for oral medications prescribed, measured as MPR, at the time of evaluation for urate-lowering (Gout), hypertension (HT), hyperlipidemia (HL), and diabetes. ${ }^{*} p<0.05$ compared to other medications

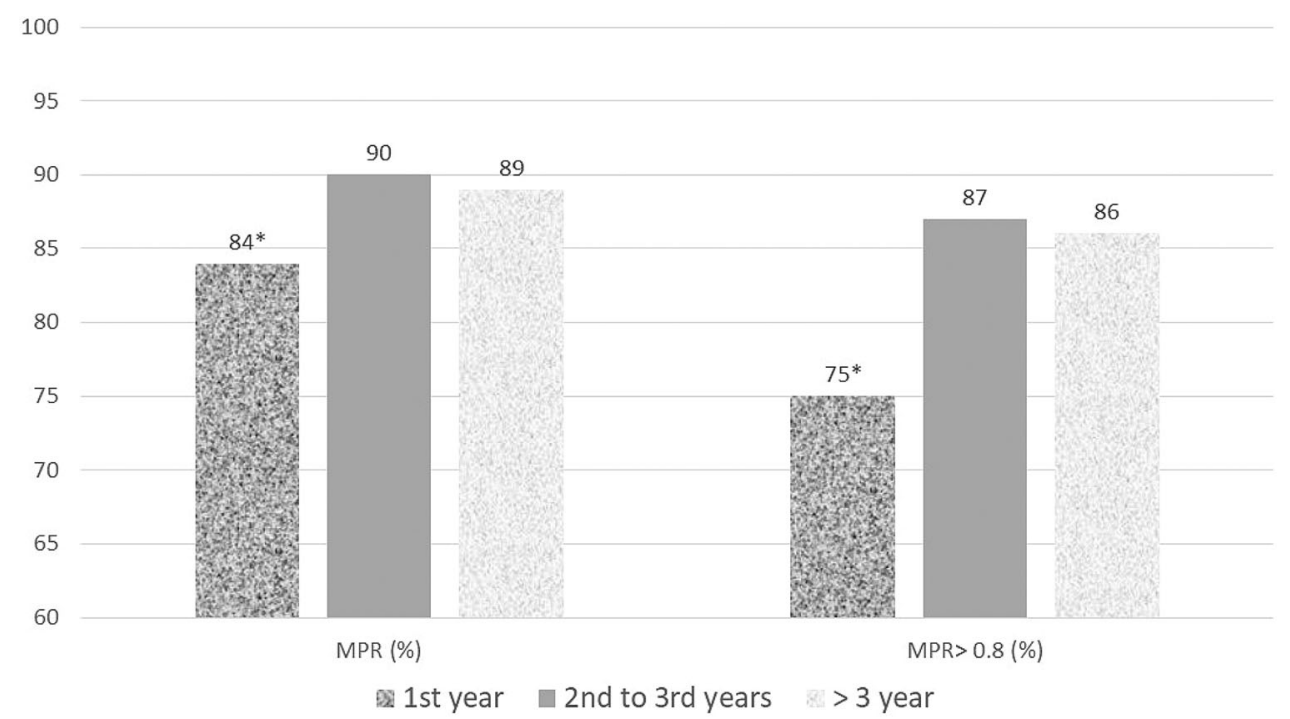

Fig. 2 Adherence for urate-lowering medication (Gout) as MPR (\%) and percentage of patients showing MPR $>0.8$ during sequential strata of follow-up time

\section{DISCUSSION}

Adherence to treatment is universally associated with optimal patient outcomes for all chronic diseases. In this prospective cohort, we demonstrate that high adherence rates are achievable in carefully managed clinical cohorts which are strongly associated with optimal achievement of sUA targets of below $6.0 \mathrm{mg} / \mathrm{dl}$.
The high rates of adherence are a consequence of several factors, as explained below in limitations, but are at least partially due to strong patient engagement and empowerment though education and suggest that the adaption of these in routine clinical practice may lead to better patient outcomes in gout.

Two recent systematic reviews and metaanalyses examining the rate of adherence in 
Table 2 Multivariable regression for adherence MPR $>0.8$

\begin{tabular}{llllr}
\hline Variables for MPR $>\mathbf{0 . 8}$ & Beta (unadjusted) & $\boldsymbol{p}$ & Beta (adjusted) & $\boldsymbol{p}$ \\
\hline Age & -0.049 & 0.497 & -0.012 & 0.850 \\
Gender (male) & -0.139 & 0.023 & -0.141 & 0.016 \\
Complexity & 0.117 & 0.063 & 0.138 & 0.019 \\
Hypertension & 0.053 & 0.451 & 0.046 & 0.500 \\
Time on follow-up & 0.026 & 0.669 & 0.060 & 0.332 \\
Loss to follow-up & -0.279 & 0.000 & -0.269 & $<0.001$ \\
\hline
\end{tabular}

Table 3 Multivariable regression analysis for achieving sUA target of below $6 \mathrm{mg} / \mathrm{dl}$

\begin{tabular}{lcccc}
\hline Variables for sUA $<\mathbf{6 ~} \mathbf{~ g} / \mathbf{d l}$ & Beta (unadjusted) & $\boldsymbol{p}$ & Beta (adjusted) & $\boldsymbol{p}$ \\
\hline Gender (male) & 0.008 & 0.906 & -0.023 & 0.737 \\
Time from onset & 0.035 & 0.665 & 0.058 & 0.394 \\
SUA at baseline & -0.045 & 0.528 & -0.017 & 0.797 \\
Polyarticular & 0.002 & 0.984 & 0.034 & 0.612 \\
CKD & 0.081 & 0.330 & 0.019 & 0.779 \\
Previous ULT & -0.007 & 0.917 & 0.013 & 0.847 \\
Previous CV event & -0.047 & 0.564 & 0.19 & 0.778 \\
Time on follow-up & 0.129 & 0.068 & 0.179 & 0.008 \\
Adherence MPR $>0.8$ & 0.191 & 0.005 & 0.225 & 0.001 \\
\hline
\end{tabular}

$s U A$ serum urate, CKD-Epi equation $<60 \mathrm{ml} / \mathrm{mIn}, C V$ cardiovascular

gout mostly coincide in reporting low adherence as MPR or proportion of days covered, with an average of $42 \%$, ranging from $17 \%$ to $83.5 \%$, $47 \%$ of patients being adherent [13], and MPR $44 \%$, ranging from $17 \%$ to $78 \%, 46 \%$ of patients being reported as adherent [14]. Therefore, adherence to treatment is to be considered poor in patients with gout.

Adherence rates differ depending on populations included: single centers and prospective cohorts show higher rates of adherence than those retrieved from administrative databases $[13,14]$. Although some authors have found no difference in adherence among countries [14], others have concluded that data from Oceania and Asia show higher adherence rates [13]. In fact, mean adherence to ULT measured with
MPR and using a health insurance database in Japan has been shown to be over $80 \%$ for allopurinol and benzbromarone [15], twofold that reported for Europe or the USA using similar databases [13].

Our data suggest that patients who are prospectively followed up show high adherence rates and close to $90 \%$ patients reach sUA therapeutic target. Poor adherence for ULT is associated with poorer clinical outcomes as sUA levels are a surrogate for gout outcomes $[16,17]$.

Different factors may influence adherence. Our patients showed a similarly high MPR for other oral medications compared with ULT. Our patients are older and commonly show multiple comorbid conditions, variables that have been described to be associated with increased 
adherence compared to otherwise healthy individuals and youngsters [18]. Other factors influencing adherence include cultural [15] and behavioral ones [19].

Our study shows that patients were persistent on follow-up with $92 \%$ of patients attending scheduled visits with a high rate of adherence, increasing during long-term followup whereas in other studies, adherence decreased with long-term prescription $[5,20]$.

We observed that taking medications for other comorbid conditions may help in increasing adherence rates, as reported by others [18]. Availability of electronic MPR, corrected for in-hospital stay, and an alert signal in the electronic prescription page may also help to avoid mistreatment and nonadherence. Loss to follow-up was associated with previous poor adherence, while good adherence and time on follow-up were strongly associated with reaching therapeutic sUA target.

Educational interventions show the highest level of evidence for improving adherence in chronic inflammatory diseases [21]. Other interventions include pharmacist-led, telemedicine, and nurse-led interventions [22]. Interventions that increase rates of adherence and therefore achievement of sUA therapeutic targets and clinical outcomes are cost-effective [9]. The initial nurse-led support has been demonstrated to be associated with high longterm adherence even despite no further followup being implemented [8], supporting the principle that adequate follow-up may improve adherence and achievement of therapeutic sUA target, as shown in this study.

Some limitations are inherent to the design of the study: there is no control group to compare to; the intervention may not be limited to educational issues but also the fact that assistance was being given in a third-level hospital setting; and finally a good MPR does not always mean that patients are taking medication, and may overestimate adherence.

\section{CONCLUSION}

Adherence rates are high in clinical cohorts empowered with modest educational support and routine follow-up. Adequate adherence is associated with continuing follow-up, the presence of comorbid conditions, and with increased rates of sUA within therapeutic target. This can be easily interpreted: empower and support patients, do not blame them, in order to achieve high rates of adherence and better patient outcomes.

\section{ACKNOWLEDGEMENTS}

We heartfully thank the participants (patients and relatives) of the study.

Funding. This work has been partially supported by a grant (FPR) from Asociación de Reumatólogos del Hospital de Cruces; no other sources of funding have been used. No Rapid Service Fee was received by the journal for the publication of this article.

Authorship. All named authors meet the International Committee of Medical Journal Editors (ICMJE) criteria for authorship for this article, take responsibility for the integrity of the work as a whole, and have given their approval for this version to be published.

Authorship Contributions. All authors shared the preliminary idea. FPR performed the statistical analysis and wrote a draft paper without any external support. All authors actively discussed results, significantly contributed to the manuscript, and approved the final version and review.

Disclosures. Fernando Perez-Ruiz: Advisor or speaker for Arthriti, Astellas, AstraZeneca, Dyve, Grünenthal, Horizon, Menarini, Syneos, Selecta. Nuria Perez-Herrero has nothing to disclose. Pascal Richette: advisor or speaker fees from Grunenthal, Ipsen Pharma, Menarini France, Astra Zeneca. Austin G. Stack: Advisor or speaker fees from Astellas, Grünental, AstraZeneca and Menarini. Unrestricted educational grant Menarini.

Compliance with Ethics Guidelines. This study was conducted in accordance with the 
Helsinki Declaration. Data were retrieved from a gout clinical database that is approved by the local ethics committee (Cruces University Hospital Ethics and Investigation Committee), with informed-signed permission from patients or relatives if appropriate.

Data Availability. The datasets generated during and/or analyzed during the current study are available from the corresponding author on reasonable request.

Open Access. This article is licensed under a Creative Commons Attribution-NonCommercial 4.0 International License, which permits any non-commercial use, sharing, adaptation, distribution and reproduction in any medium or format, as long as you give appropriate credit to the original author(s) and the source, provide a link to the Creative Commons licence, and indicate if changes were made. The images or other third party material in this article are included in the article's Creative Commons licence, unless indicated otherwise in a credit line to the material. If material is not included in the article's Creative Commons licence and your intended use is not permitted by statutory regulation or exceeds the permitted use, you will need to obtain permission directly from the copyright holder. To view a copy of this licence, visit http://creativecommons.org/licenses/by$\mathrm{nc} / 4.0 /$.

\section{REFERENCES}

1. FitzGerald JD, Dalbeth N, Mikuls T, et al. 2020 American College of Rheumatology guideline for the management of gout. Arthritis Care Res (Hoboken). 2020;72(6):744-60.

2. Perez-Ruiz F, Desideri G. Improving adherence to gout therapy: an expert review. Ther Clin Risk Manag. 2018;14:793-802.

3. Hill-McManus D, Marshall S, Soto E, Lane S, Hughes D. Impact of non-adherence and flare resolution on the cost-effectiveness of treatments for gout: application of a linked pharmacometric/pharmacoeconomic model. Value Health. 2018;21(12):1373-81.
4. Coleshill MJ, Aung E, Nguyen AD, et al. Improving adherence to urate-lowering therapy in people living with gout. Int J Rheum Dis. 2019;22(4):542-4.

5. Mantarro S, Capogrosso-Sansone A, Tuccori M, et al. Allopurinol adherence among patients with gout: an Italian general practice database study. Int J Clin Pract. 2015;69(7):757-65.

6. Vaccher S, Kannangara DR, Baysari MT, et al. Barriers to care in gout: from prescriber to patient. J Rheumatol. 2016;43(1):144-9.

7. Abhishek A, Doherty M. Education and non-pharmacological approaches for gout. Rheumatology (Oxford). 2018;57(suppl_1):i51-8.

8. Abhishek A, Jenkins W, La-Crette J, Fernandes G, Doherty $M$. Long-term persistence and adherence on urate-lowering treatment can be maintained in primary care-5-year follow-up of a proof-of-concept study. Rheumatology (Oxford). 2017;56(4):529-33.

9. Doherty M, Jenkins W, Richardson H, et al. Efficacy and cost-effectiveness of nurse-led care involving education and engagement of patients and a treatto-target urate-lowering strategy versus usual care for gout: a randomised controlled trial. Lancet. 2018;392(10156):1403-12.

10. Richette P, Doherty M, Pascual E, et al. 2016 updated EULAR evidence-based recommendations for the management of gout. Ann Rheum Dis. 2017;76(1):29-42.

11. Antiplatelet Trialists' Collaboration. Collaborative overview of randomised trials of antiplatelet therapy-I: Prevention of death, myocardial infarction, and stroke by prolonged antiplatelet therapy in various categories of patients. BMJ. 1994;308(6921): 81-106.

12. Besga A, Ayerdi B, Alcalde G, et al. Risk factors for emergency department short time readmission in stratified population. Biomed Res Int. 2015;2015: 685067.

13. Yin R, Li L, Zhang G, et al. Rate of adherence to urate-lowering therapy among patients with gout: a systematic review and meta-analysis. BMJ Open. 2018;8(4):e017542.

14. Scheepers L, van Onna M, Stehouwer CDA, Singh JA, Arts ICW, Boonen A. Medication adherence among patients with gout: a systematic review and meta-analysis. Semin Arthritis Rheum. 2018;47(5): 689-702.

15. Koto R, Nakajima A, Horiuchi H, Yamanaka H. Realworld treatment of gout and asymptomatic hyperuricemia: a cross-sectional study of Japanese health 
insurance claims data. Mod Rheumatol. 2020. https://doi.org/10.1080/14397595.2020.1784556.

16. Stamp L, Morillon MB, Taylor WJ, et al. Serum urate as surrogate endpoint for flares in people with gout: a systematic review and meta-regression analysis. Semin Arthritis Rheum. 2018;48(2):293-301.

17. Perez-Ruiz F, Martin I, Canteli B. Ultrasonographic measurement of tophi as an outcome measure for chronic gout. J Rheumatol. 2007;34(9):1888-93.

18. De Vera MA, Marcotte G, Rai S, Galo JS, Bhole V. Medication adherence in gout: a systematic review. Arthritis Care Res (Hoboken). 2014;66(10):1551-9.

19. Reach G. Treatment adherence in patients with gout. Jt Bone Spine. 2011;78(5):456-9.

20. Janssen CA, Oude Voshaar MAH, Vonkeman HE, Krol M, van de Laar M. A retrospective analysis of medication prescription records for determining the levels of compliance and persistence to uratelowering therapy for the treatment of gout and hyperuricemia in the Netherlands. Clin Rheumatol. 2018;37(8):2291-6.

21. Lavielle M, Puyraimond-Zemmour D, Romand X, et al. Methods to improve medication adherence in patients with chronic inflammatory rheumatic diseases: a systematic literature review. RMD Open. 2018;4(2):e000684.

22. Gill I, Dalbeth N, Ofanoa M, Goodyear-Smith F. Interventions to improve uptake of urate-lowering therapy in patients with gout: a systematic review. BJGP Open. 2020;4(3). https://doi.org/10.3399/ bjgpopen20X101051. 